\title{
Effect of age on contents of fatty acids in whole bodies of pheasants throughout their growth
}

\author{
Petra Jakešová1, David Zapletal ${ }^{1}$, Radovan Jůzl ${ }^{1}$, Lucie Rusníková ${ }^{2}$, Pavel Suchý1, \\ Eva Straková ${ }^{2}$
}

University of Veterinary and Pharmaceutical Sciences Brno, Faculty of Veterinary Hygiene and Ecology, ${ }^{1}$ Department of Animal Husbandry and Animal Hygiene, ${ }^{2}$ Department of Animal Nutrition, Brno, Czech Republic

Received November 28, 2013

Accepted March 26, 2014

\begin{abstract}
The aim of the work was to evaluate the effect of age on the proportion of particular fatty acids in the whole bodies of pheasants fattened for up to 118 days of age. In the experiment, 232 chicks of common pheasants (Phasianus colchicus) were used. In the course of the experiment, the required number of pheasants was randomly selected for analysis at regular 10-day intervals. The age of pheasant chicks had a significant effect on the content of all fatty acids. Significant changes in the proportion of fatty acid groups were recorded mainly at 20,40 and 60 days of age. By 20 days of age the proportion of monounsaturated fatty acids decreased considerably in relation to the increase in the content of polyunsaturated fatty acids, while the $\mathrm{n} 6 / \mathrm{n} 3$ ratio decreased markedly. The content of monounsaturated fatty acids increased between day 20 and day 40, causing a simultaneous decrease in polyunsaturated fatty acids. From day 40 , the monounsaturated fatty acid content increased gradually till the end of fattening, whereas the reverse trend was observed in the saturated fatty acid content in this period. As for polyunsaturated fatty acids, their presence increased from day 40 to day 60 , followed by a gradual decrease till the end of fattening. The results of our work showed the different ability of pheasants to digest and utilize particular fatty acids with varying efficiency throughout their growth. The study brings new findings concerning changes in fatty acid composition of pheasants during fattening.
\end{abstract}

Feathered game, fat composition, fattening, 10-day period

Game meat is a good source of proteins, fatty acids, vitamins and trace elements (Nuernberg et al. 2009). Pheasant meat in particular, thanks to the high content of some proteins and the low content of fat that moreover shows a higher proportion of essential fatty acids (FA), is a highly valued food, outdoing even the nutritional value of broiler chicken meat (Straková et al. 2006; Straková et al. 2011; Vitula et al. 2011).

Poureslami et al. (2010a) found that the effect of age on the proportions of FA in broiler chickens was only negligible. On the other hand, Poureslami et al. (2010b) discovered an increase in elongase activity as broiler chickens aged. The effect of age on the digestibility of FA in broiler chickens was studied by Zelenka et al. (2003). These authors concluded that the chicken age itself had a highly demonstrable role in the digestibility of saturated fatty acids (SFA), monounsaturated fatty acids (MUFA) and polyunsaturated fatty acids (PUFA). In addition, as regards the composition of pheasant fat, Sarra et al. (1985) found that the age of pheasants was associated with significant differences in the muscle lipid composition of different anatomical locations. However, the targeted evaluation of the FA content in the pheasant body throughout growth has not yet been studied by anyone in the available literature.

Therefore, the aim of our work was to evaluate the effect of the age of pheasant chicks on the contents of particular fatty acids and their groups in whole pheasant bodies fattened by the age of 118 days. 


\section{Materials and Methods}

The study was carried out in an accredited experimental stable of the Department of Animal Nutrition at the UVPS Brno with controlled housing conditions 63/2008. A total of 232 chicks of common pheasants (Phasianus colchicus) were used in the experiment; housed on a deep litter floor. Pheasants were housed in floor pens (area $\left.7 \mathrm{~m}^{2} / \mathrm{pen}\right)$, in which protective chick guards were placed for the first 14 days. The temperature regime at the start of the experiment was $31{ }^{\circ} \mathrm{C}$; gradually the temperature was reduced by $2{ }^{\circ} \mathrm{C}$ a week to a temperature of $21{ }^{\circ} \mathrm{C}$. The temperature

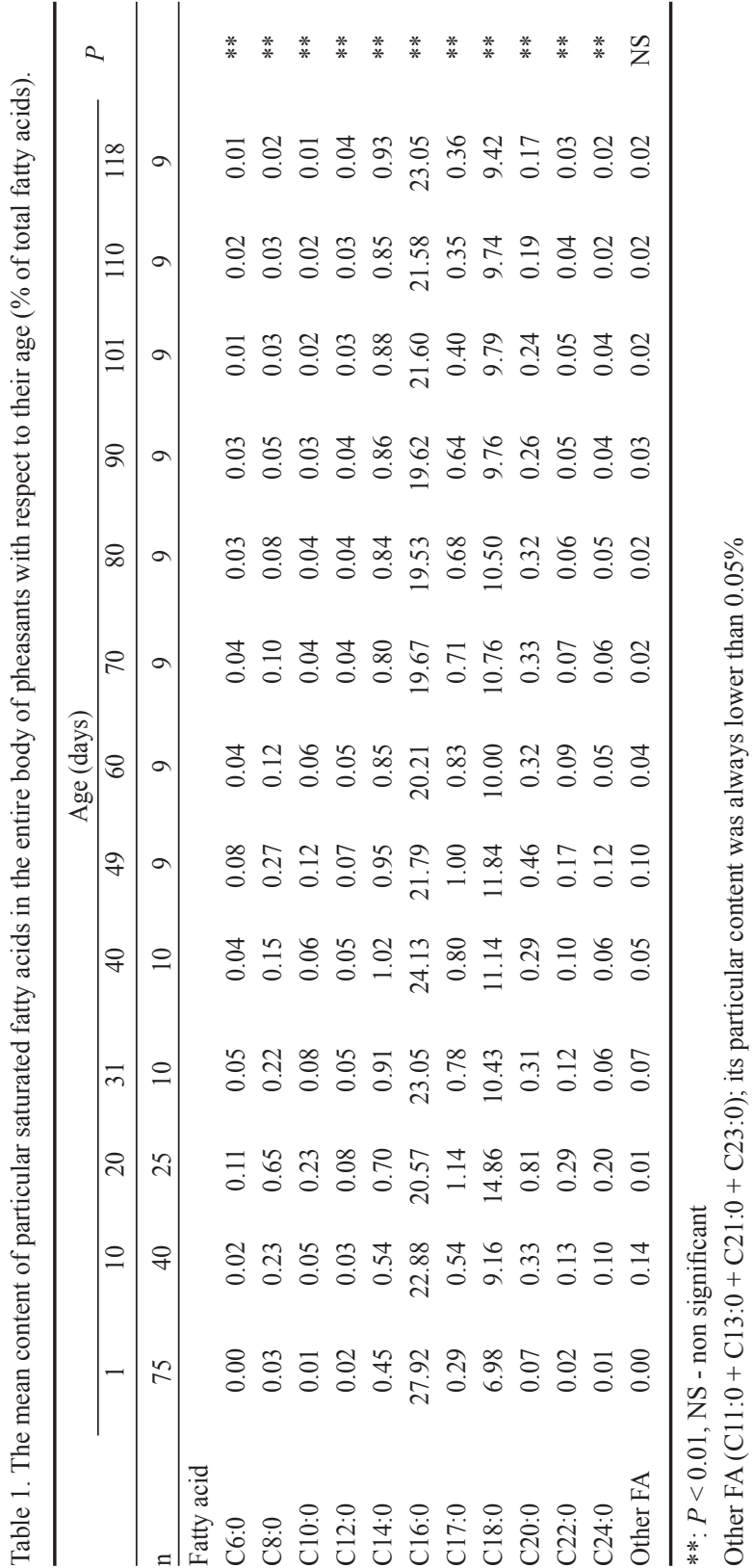
was maintained using electronic henlike devices providing radiant heat. The lighting regime was the same throughout the fattening period, i.e. $23 \mathrm{~h}$ of light and $1 \mathrm{~h}$ of dark. The pheasants were fed ad libitum.

A 3-phase feeding program (starter, grower, and finisher) was used. The starter (CP - crude protein $208.9 \mathrm{~g} \cdot \mathrm{kg}$ ${ }^{1}, \mathrm{BE}$ - brutto energy $16.8 \mathrm{MJ} \cdot \mathrm{kg}^{-1}$ ) was fed to chicks till the age of 15 days, the grower (CP - $193.0 \mathrm{~g} \cdot \mathrm{kg}^{-1}, \mathrm{BE}-17.0$ $\left.\mathrm{MJ} \cdot \mathrm{kg}^{-1}\right)$ was fed from 16 to 30 days of age and the finisher (CP $-229.3 \mathrm{~g} \cdot \mathrm{kg}^{-1}$, $\mathrm{BE}-17.4 \mathrm{MJ} \cdot \mathrm{kg}^{-1}$ ) was fed until the end of the fattening period.

In the course of the experiment, the required number of pheasants was selected at random at 10-day intervals (on days $1,10,20,31,40,49,60,70,80$, $90,101,110$, and 118 of age). Within the scope of samplings no. 1 to 4 , always 5 groups (samples) out of the total number of pheasant chicks used for analyses were subdivided. To be specific, 75 chicks were used in sampling 1 (day 1 of age), 40 chicks in sampling 2, 25 chicks in sampling 3, 10 chicks in sampling 4 (day 31 of age). In sampling 5 (day 40 of age), 10 pheasants in 1 individual sample were used. Starting with sampling 6 (day 49 of age), 9 individual samples were taken. Pheasants used in all samplings were always a group consisting of both sexes that were randomly selected.

After one-day fasting, pheasants were stunned by a blow to the head and killed by bleeding. Thereafter the whole pheasant bodies (including all feathers, skin, viscera and blood) were homogenized. After sample homogenization, water was separated; fat was extracted and then esterified to prepare a sample for analysis according to Hara and Radin (1978). The extracted fat $(80 \mathrm{ml})$ was transformed to esters, and concentrations of fatty acids in the form of methyl-esters (FAMEs) were specified by gas chromatography, analyzer GC-2010 (Shimadzu, Japan) with a flame ionization detector (FID). The adopted method used the addition of an internal standard (C15:0). The GC2010 system was equipped with an auto sampler and auto injector. The injection volume was $1 \mu \mathrm{l}$. The total split flow 
was $90 \mathrm{ml} / \mathrm{min}$. Helium was used as the carrier gas. Air and hydrogen were used as auxiliary gases. The FAMEs were separated on a VB-WAX column ( $60 \mathrm{~m}$ length; $0.25 \mathrm{~mm}$ ID; $0.25 \mu \mathrm{m}$ film thickness). The FID temperature was set at $300{ }^{\circ} \mathrm{C}$, initial injector temperature was $280^{\circ} \mathrm{C}$ and pressure was $299.2 \mathrm{kPa}$; initial column temperature was $70{ }^{\circ} \mathrm{C}$. The contents of fatty acids are expressed in $\%$ of total fatty acids in samples.

Statistical analysis was performed using Statistica CZ, version 10. ANOVA analysis was used to study the differences in fatty acid composition and the ratios of the whole pheasant body as thirteen independent groups according to age;

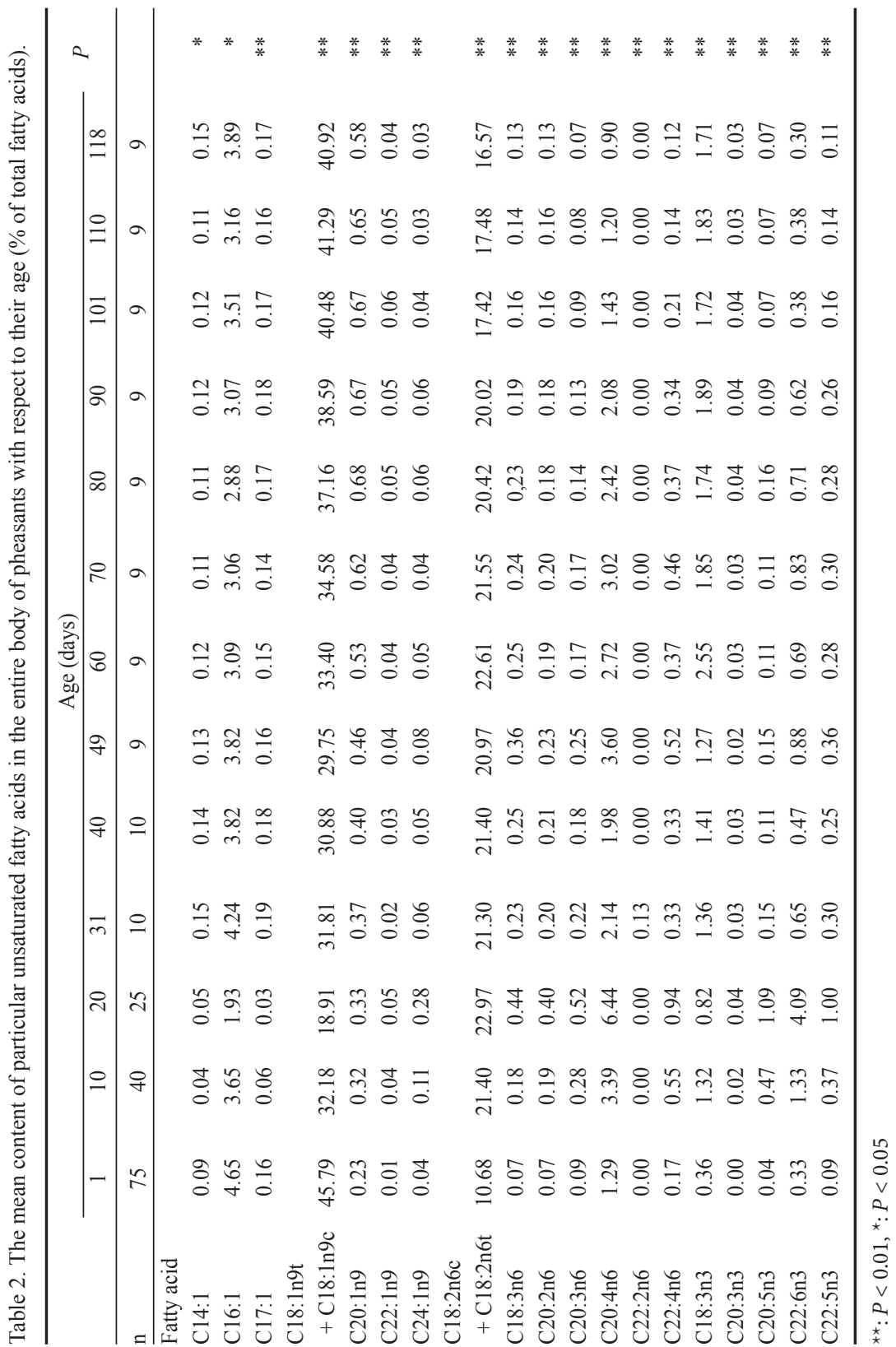


while the age was the single factor included in a statistical model. When the analysis of variance showed significant differences between the groups, Sheffe's test was used.

\section{Results}

The age of pheasant chicks had a significant effect on the content of all SFA (Table 1). The highest content of palmitic acid (C16:0) was found on day 1 of age, its content thereafter

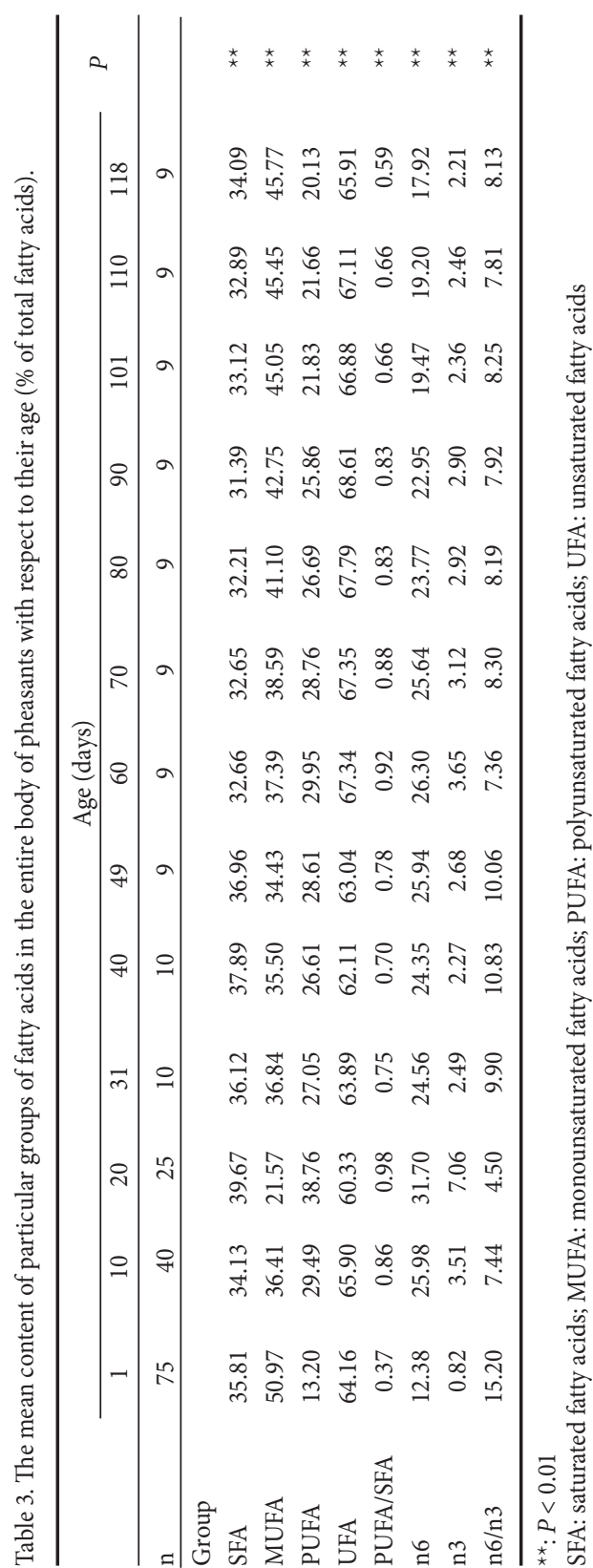
decreased through day 20 of age $(P<$ $0.01)$, subsequently culminated on day $40(P<0.05)$, followed by a decrease on day 90 . At the end of fattening its content had increased $(P<0.01)$. A considerable increase in the stearic acid (C18:0) content occurred on days 20 and 40 of age $(P<$ 0.01 ), followed by a gradual decrease towards the end of fattening. In the course of the evaluated period, the same trend was observed for the proportion of caproic acid (C6:0), caprylic acid (C8:0), capric acid (C10:0), lauric acid (C12:0), margaric acid (C17:0), arachidic acid (C20:0), behenic acid (C22:0) and lignoceric acid (C24:0). The contents of these FA increased until day $20(P<0.01)$, then decreased through day 40, and increased again on day $49(P$ $<0.01)$, whereupon the contents decreased gradually till the end of fattening.

Table 2 indicates that pheasant age had a significant effect on contents of all MUFA. The content of elaidic + oleic acid $(\mathrm{C} 18: \ln 9 \mathrm{t}+\mathrm{C} 18: \ln 9 \mathrm{c})$ was highest on day 1 of age, then it demonstrably decreased till day 20 of age after which it significantly increased till the end of the experiment. Similarly to $\mathrm{C} 18 \mathrm{~s} \ln 9 \mathrm{t}$ $+\mathrm{C} 18: 1 \mathrm{n} 9 \mathrm{c}$, the highest content of palmitoleic acid (C16:1) was found on day 1 of age with a subsequent minimum on day $20(P<0.05)$; thereafter there was an increase on day 31 , followed by a decrease between days 60 and 90 of fattening. The content of gondoic acid (C20:1n9) increased from day 1 to day $80(P<0.01)$, thereafter it decreased slightly till day 118 . The proportion of erucic acid (C22:1n9) and nervonic acid (C24:1n9) significantly rose to day 20, after which their proportions decreased till day 40 and increased on day 49; then the content of $\mathrm{C} 22: \ln 9$ did not change significantly and the content of C24:1n9 
significantly decreased at the end of fattening. In the case of myristoleic acid (C14:1) and ginkgolic acid (C17:1), their content decreased on day 20, a significant increase followed on day 40, whereupon their contents remained almost unchanged for the rest of fattening.

The lowest presence of linoleic + linolelaidic acids (C18:2n6c $+\mathrm{C} 18: 2 \mathrm{n} 6 \mathrm{t}$, respectively) was found on day 1 of age $(P<0.01)$, their contents increased rapidly as early as on day 10 and remained at a similar level till day 90 of age, after which they decreased slightly at the end of fattening. Arachidonic (C20:4n6), adrenic (C22:4n6), eicosapentaenoic (C20:5n3), docosahexaenoic (C22:6n3) and docosapentaenoic (C22:5n3) acids underwent the same trend for proportion in whole pheasant bodies throughout the evaluated period. Contents of these FA showed an increase on day $20(P<0.01)$, with a subsequent decrease on day 40 , a demonstrable increase on day $49(P<0.01)$ and a subsequent gradual decrease till the end of fattening. The same trend was seen in the contents of $\gamma$-linolenic $(\mathrm{C} 18: 3 \mathrm{n} 6)$, eicosadienoic (C20:2n6) and mono-g-linolenic (C20:3n6) acids. The presence of $\alpha$-linolenic acid $(\mathrm{C} 18: 3 \mathrm{n} 3)$ was the lowest on day 1 of age $(P<0.01)$; from day 10 it increased with a subsequent culmination on day $60(P<0.01)$; thereafter its content remained almost unchanged till the end of fattening.

Significant changes in the proportion of FA groups were seen mainly on day 20, 40 and 60 of chicks' age (Table 3). Up until day 20 of age the proportion of MUFA decreased considerably, which also related to the considerable increase in the PUFA content, while the $\mathrm{n} 6 / \mathrm{n} 3$ ratio decreased markedly $(P<0.01)$. In addition, the SFA content increased only slightly and from day 20 to day 40 it remained virtually unchanged. Compared to the previous period, MUFA contents increased, which conversely caused a reduction in proportion of PUFA, while the n6/n3 ratio increased considerably $(P<0.01)$. From day 40, the MUFA content increased gradually till the end of fattening $(P<0.01)$, whereas the reverse trend occurred in the SFA proportion (except for day 118). The PUFA content increased from day 40 to 60 with a subsequent constant gradual decrease till the end of fattening. The n6/n3 ratio decreased slightly till day 60 and thereafter did not change significantly.

\section{Discussion}

Komprda et al. (2002) found in turkey meat that as the MUFA content increased with age, the PUFA content decreased. This corresponds to the results of our study. Similarly, Poureslami et al. (2010a) stated that the content of PUFA n3 and n6 in various parts of broiler chickens decreased with their age. On the other hand, Baeza et al. (2000) found in duck meat that age did not have an effect on contents of SFA and MUFA up to 90 days of age.

Zelenka et al. (2003) found that digestibility of all SFA and MUFA (with the exception of C20:1 n-9) decreased demonstrably from day 9 to day 42 of age in broiler chickens, whereas in this period digestibility of PUFA was higher than that of both SFA and MUFA. These findings correspond to some degree to our results for FA content till day 20 of pheasant chicks, despite the fact that there was a change in the feed on day 15 of age. Moreover, it is apparent that also the $2^{\text {nd }}$ change of feed, on day 30 of age, did not have any fundamental long-term effect on trends in the deposition of particular FA groups in whole pheasant bodies. This indicates the different ability of pheasants to digest and utilize particular FA with varying efficiency at different ages.

The age of pheasants had a significant effect on the contents of all FA. Results of this study reveal that significant changes in the proportion of FA groups were seen on days 20, 40 and 60 of age. Our results indicate the varying ability of pheasants to digest and utilize particular FA with varying efficiency throughout their growth. 


\section{Acknowledgement}

This study was funded by the grant IGA 31/2013/FVHE.

\section{References}

Baeza E, Salichon MR, Marche G, Wacrenier N, Dominguez B, Culioli J 2000: Effects of age and sex on the structural, chemical and technological characteristics of mule duck meat. Brit Poultry Sci 41: 300-307

Hara A, Radin NS 1978: Lipid extraction of tissues with a low-toxicity solvent. Anal Biochem 90: 420-426

Komprda T, Šarmanová I, Zelenka J, Bakaj P, Fialová M, Fajmonová E 2002: Effect of sex and age on cholesterol and fatty acid content in turkey meat. Arch Geflugelkd 66: 263-273

Nuernberg K, Numberg G, Dannenberger D 2009: Nutrient and lipid composition of muscle in wild animals. Fleischwirtschaft 89: 99-102

Poureslami R, Raes K, Huyghebaert G, De Smet S 2010a: Effects of diet, age and gender on the polyunsaturated fatty acid composition of broiler anatomical compartments. Brit Poultry Sci 51: 81-91

Poureslami R, Turchini GM, Raes K, Huyghebaert G, De Smet S 2010b: Effect of diet, sex and age on fatty acid metabolism in broiler chickens: SFA and MUFA. Brit J Nutr 104: 204-213

Sarra C, Boccignone M, Damasio L 1985: The Effect of age, sex, and anatomical location on the fatty acid composition of pheasant meat. Poultry Sci 64: 1090-1097

Straková E, Suchý P, Karásková K, Jámbor M, Navrátil P 2011: Comparison of nutritional values of pheasant and broiler chicken meats. Acta Vet Brno 80: 373-377

Straková E, Suchý P, Vitula F, Večerek V 2006: Differences in the amino acid composition of muscles from pheasant and broiler chickens. Arch Tierzucht 49: 508-514

Vitula F, Suchý P, Straková E, Karásková K, Zapletal D, Kroupa L 2011: Energy value of meat in selected species of feathered game. Acta Vet Brno 80: 197-202

Zelenka J, Fajmonová E, Kladroba D 2003: Effect of age on digestibility of fatty acids in chickens. Czech J Anim Sci 48: $315-320$ 